\title{
Coherent backscattering of light in a strong localization regime
}

\author{
Xiangdong Zhang and Zhao-Qing Zhang \\ Department of Physics, The Hong Kong University of Science and Technology, Clear Water Bay, Kowloon, Hong Kong, China
}

(Received 1 June 2001; revised manuscript received 26 November 2001; published 4 April 2002)

\begin{abstract}
Coherent backscattering (CBS) of light in a strong localization regime is studied with absorption/ amplification included. The CBS cones arising from strongly localized states inside the complete gaps of disordered photonic crystals have been observed by numerical simulations in two dimensions. The structure of the CBS cones can be described well by a theory that incorporates mean free paths, localization length, amplification/absorption length, and single-particle scattering anisotropy. The excellent agreement between theory and simulations without any adjustable parameters makes it possible to determine the localization length from a measured CBS cone, even in an absorbing medium.
\end{abstract}

DOI: 10.1103/PhysRevB.65.155208

PACS number(s): 42.25.Dd, 42.25.Hz

\section{INTRODUCTION}

During the past decade the localization of classical waves in random media has been the subject of intensive studies. ${ }^{1-13}$ The observation of light localization has been reported based on the exponential decay of light intensity as it propagates through the media. ${ }^{2,3}$ However, these reports have come under close scrutiny because of the presence of absorption in the media. How to probe localized states in absorbing media has become a crucial issue at present. Recently, a statistical approach has been taken to determine the extent of localization in the presence of absorption. ${ }^{4}$ In addition to transmission measurements, one may ask the question: Is it possible to detect localization from reflection measurements? Recent results based on random matrix theory indicate that the statistics of reflected intensity cannot provide localization information. ${ }^{5}$ However, it is well known that the structure of coherent backscattering (CBS) cones has provided a useful means to estimate the mean free path of the system in the diffusive regime. ${ }^{6,7}$ A natural question to ask is whether the structure of the CBS cone in a localized regime can provide localization information of the system as well.

The phenomenon of enhanced backscattering, a precusor of Anderson localization due to the interference effect, has been well studied in weak scattering media. ${ }^{6,7}$ In the presence of absoprtion (amplification), the CBS cone becomes rounded (sharper) due to the suppression (enhancement) of long optical paths. ${ }^{7-9}$ However, the theoretical study of the structure of the CBS cone in the critical region close to the localization transition has been an interesting and challenging problem. ${ }^{10-13}$ In this region, the structure of the CBS cone can change considerably due to the renormalization of the diffusion constant. Just above the localization transition, it is expected that the CBS cone has a sharp top with a round region close to it. Just below the localization transition, the sharp top disappears and a rounding of the CBS cone appears. ${ }^{10,11,13}$ The rounding of the CBS cone has been observed near the critical region. ${ }^{12}$ In Ref. 10 , the authors consider a momentum-space renormalization of the diffusion constant $D(q)$. Due to the requirement of flux conservation, the authors also assume that the boundary layers at the surface (or extrapolation length) should scale in the same way as the diffusion constant to avoid artificial logarithmic diver- gence in the total reflection. However, this assumption is not made in the study of Ref. 11. Very recently, a self-consistent theory has been proposed to describe CBS near the mobility edge in the absence of absorption. ${ }^{13}$ In this work, the authors take an approach of renormalizing the diffusion constant in real space. Again, no extrapolation length renormalization is assumed. Thus, whether or not an extrapolation length renormalization is necessary remains a controversial issue.

Much attention has been paid to the critical region, but little attention has been paid to the structure of the CBS cone in the strong localization regime far below the Anderson localization transition, which is actually an interesting problem in its own right. One may ask the following question: If the localization length is so small that it is of the order of the "lattice constant," would there still be a CBS cone? If so, could its structure provide any useful localization information? In fact, Ref. 10 has predicted a totally flat curve with no CBS cone in the strong localization regime. With the rapid development of the photonic band-gap materials, it has become more likely to achieve strong localization states inside the complete gap of a disordered photonic crystal. ${ }^{14,15}$ The observation of strongly localized states has been reported in both two ${ }^{16}$ and three dimensions. ${ }^{17,18}$ Recently, CBS measurements have been performed at frequencies inside the incomplete gaps of weakly disordered photonic crystals. However, only diffusive behavior was found. ${ }^{19}$ Even in random media, localized states with a localization length equal to ten times that of mean free path have been achieved recently, ${ }^{4}$ which is not far from the strong localization regime considered here.

In this work, by using the multiple-scattering method, we study the structure of CBS cones arising from strongly localized states inside the complete gap of disordered photonic crystals in two dimensions. ${ }^{20}$ This method is known to accurately reproduce experimental measurements. Through this simulation, we find that the CBS cone still exists and the curve is not flat even when the localization length $\xi$ is two to three times that of the lattice constant, contrary to the previous prediction in Ref. 10. What is more interesting is that, from the results of three different wavelengths and localizations lengths, we find that the rounding of the CBS cone follows a simple relation $\lambda / \xi$, where $\lambda$ is the wavelength. In the absence of gain or absorption, such a relation makes the 
determination of localiztion length possible. In the presence of gain or absorption, we find that the CBS cone becomes sharper or rounded as it does in the diffusive regime.

In addition to simulation, in this work we also present a theoretical approach to study the CBS cone in the strong localization regime. Our approach is similar to that described in Ref. 10. However, we incorpate wave localization in a slightly different way. In addition, we consider the singleparticle scattering contribution to the CBS cones, which is found to be important in the strong localization regime. The effects due to absorption/amplification in the medium are also included in our approach. Thus, our theory incorporates scattering and transport mean free paths, localization length, absorption/amplification length, and single-particle scattering anisotropy. Due to strong wave localization, the issue of extrapolation length renormalization becomes unimportant. Our theory is capable of quantitatively reproducing the simulation data for a wide range of angles in all the cases we have studied without any adjustable parameters. The excellent agreement between theory and simulation has made it possible to determine the localization length from a measured CBS cone, even in the presence of absorption. An analytical result for three dimensions is also presented. ${ }^{21}$

This rest of this paper is arranged as follows. In Sec. II, we first present the results of the numerical simulation. The analytical approach to the study of CBS cones is described in Sec. III. Section IV includes the discussion of our results and our conclusions.

\section{NUMERICAL SIMULATION}

We obtain a random medium by completely randomizing an ordered photonic crystal, which consists of dielectric cylinders with a radius $R$ and a dielectric constant $\epsilon=11.4$ arranged in a square lattice with air as the background. If we let $a$ denote the lattice constant, complete gaps exist for a certain range of $R / a$ for $s$-polarized waves. ${ }^{15}$ The Maxwell equation takes the form $\left[c^{-2} \omega^{2} \epsilon(\vec{r})+\nabla^{2}\right] E(\vec{r})=0$, where $E(\vec{r})$ is the electric field along the cylinder axis and $\epsilon(\vec{r})$ is the position-dependent dielectric constant.

To randomize a sample of size $W \times L$ ( $W$ layers in width and $L$ layers in thickness), we randomly move the position of each cylinder within a distance $(a-2 R) / 2$. The procedure is repeated 1000 times to ensure complete randomization. A move is forbidden if two cylinders overlap. To calculate the transmission and reflection properties of the sample, we use the multiple-scattering method described in Ref. 20. The source is prepared by passing a plane wave through an open slit in front of the sample. The width of the slit is about $20 \%$ smaller than the sample width to avoid diffraction. The multiple-scattering method allows us to calculate the nearand far-zone scattered field in both transmission and reflection geometries. ${ }^{20}$ In our calculations, we choose $W=101$ and higher freqency gaps to ensure sufficient angular resolution for the study of the CBS cone. For an ordered system, the transmission coefficients $T$ as a function of renormalized frequency $f(=\omega a / 2 \pi c)$, for a sample of size $101 \times 7$ along the $\Gamma-X$ direction are plotted in Fig. 1 for $R=0.28 a, 0.3 a$, and $0.32 a$, with dashed, dotted, and solid curves, respec-

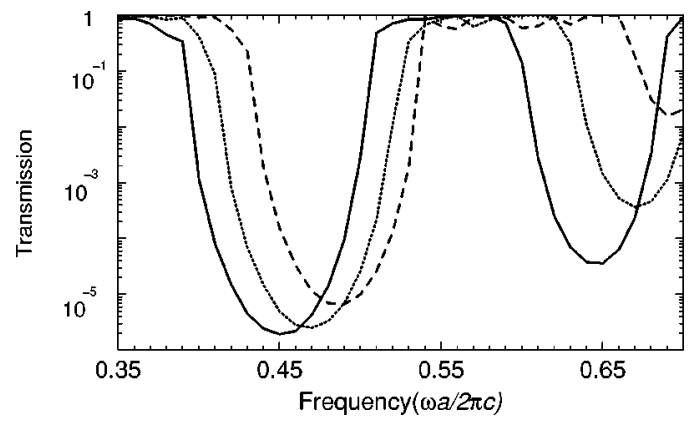

FIG. 1. $S$-wave transmission coefficients for photonic crystals in square lattice with dielectric cylinders of radii $R=0.28 a$ (the dashed line), $0.3 a$ (the dotted line), and $0.32 a$ (the solid line), and $\varepsilon=11.4$.

tively. Here, only the second and the third gaps are shown. For each value of $R$, we choose one frequency to study. The following are the three cases we have studied here: (a) $R$ $=0.30 a, f=0.48, \quad(b) \quad R=0.28 a, f=0.48$, and (c) $R$ $=0.32 a, f=0.64$. Since the chosen frequencies are near the transimssion minima as shown in Fig. 1, they all lie inside the second and the third complete gaps of the ordered systems. ${ }^{15}$ After randomization, localized states will appear inside these gaps. To determine the localization length, we take the geometric mean of the transmission coefficient, which is expected to decay exponentially with sample thickness, i.e., $T \sim \exp (-L / \xi)$ or $\langle\ln T\rangle \sim-L / \xi$. After an average of 100 configurations, we show $\langle\ln T\rangle$ as a function of sample thickness in Fig. 2(a). From the slope we find $\xi$ $=1.95 a, 3.2 a$ and $5.8 a$ for cases (a), (b), and (c), respectively. Thus, these states are indeed strongly localized with the localization length of the order of the lattice constant.

For strongly localized states, the scattering mean free path is also very small. We can simulate the scattering mean free path from the exponential attenuation of the coherent part of the transmitted field, i.e., $|\langle E\rangle|^{2} \sim \exp \left(-L / l_{s}\right){ }^{22}$ For each configuration, we measure the field at ten different points in the central speckle. Similar data are taken for 500 configurations for averaging. The results are plotted in Fig. 2(b), from which we find $l_{s}=0.56 a, 0.91 a$, and $1.28 a$ for cases (a), (b), and (c), respectively. In the presence of absorption or
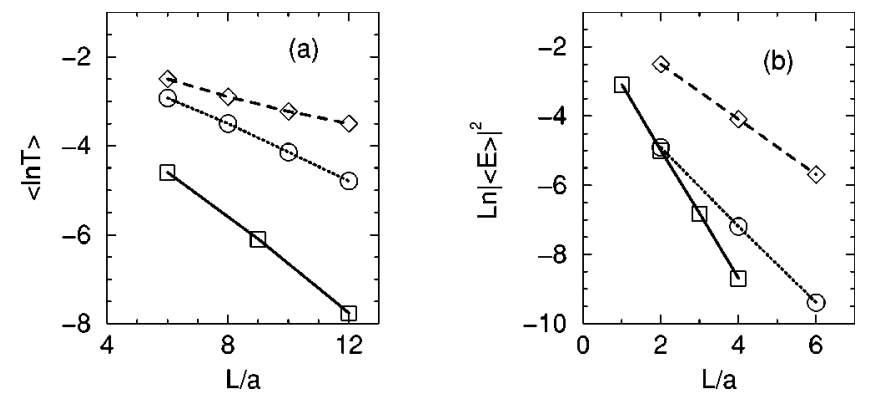

FIG. 2. The transmission coefficient, $\langle<\ln T\rangle$, as a function of sample thickness $L / a$ for three cases studied in the text: (i) $R$ $=0.3 a$ at $\omega a / 2 \pi c=0.48$ (solid line), (ii) $R=0.28 a$ at $\omega a / 2 \pi c$ $=0.48$ (dotted line), and (iii) $R=0.32 a$ at $\omega a / 2 \pi c=0.64$ (dashed line). (b) The coherent part of the transmitted field $\ln |\langle E\rangle|^{2}$ as a function of sample thickness $L / a$ for the corresponding cases in (a). 

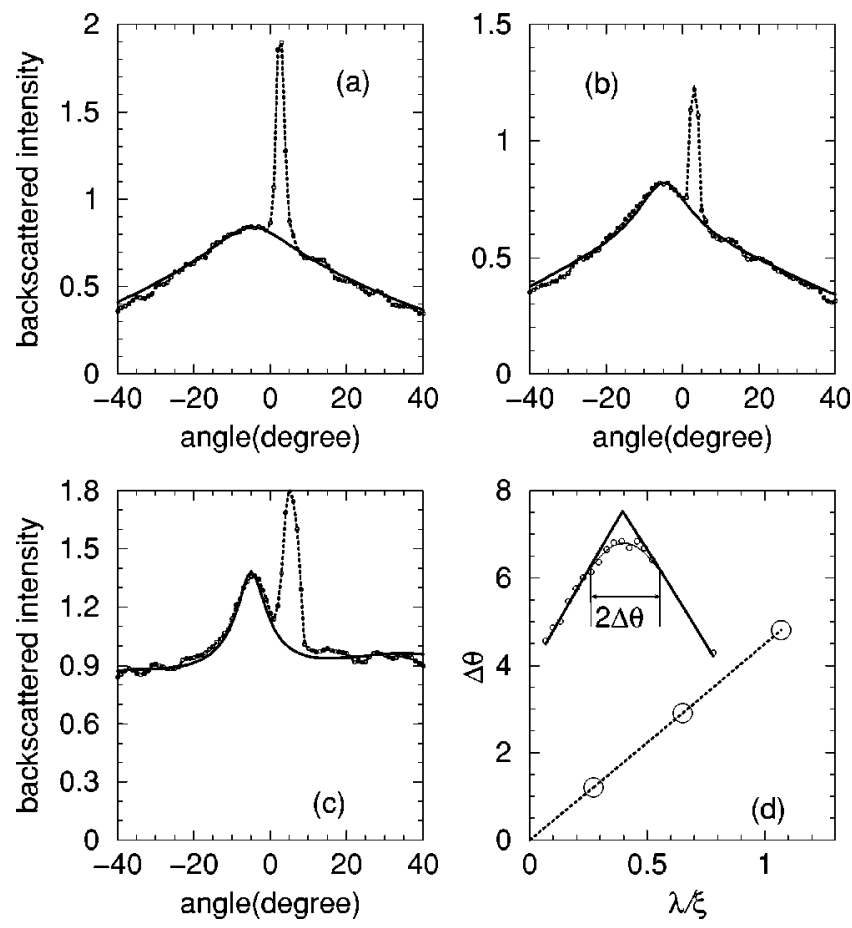

FIG. 3. Comparison between theory (solid curves) and simulations (dotted curves) of the CBS cones for three cases: (a) $R$ $=0.3 a$ at $\omega a / 2 \pi c=0.48$, (b) $R=0.28 a$ at $\omega a / 2 \pi c=0.48$, (c) $R$ $=0.32 a$ at $\omega a / 2 \pi c=0.64$. (d) shows the rounding angle of CBS cone $(\Delta \theta)$ as a function of $\lambda / \xi$. The inset shows how the degree of rounding is measured for case (a).

amplification, $|\langle E\rangle|^{2}$ decays as $\exp (-L / \bar{l})$, where $\bar{l}$ is the extinction length and is related to the absorption length $l_{a}$ or gain length $l_{g}$ by the relation $1 / \bar{l}=1 / l_{s} \pm 1 / l_{a, g}{ }^{8,9}$ The "bare" transport mean free path $l_{t}^{o}$ is related to the scattering mean free path through the relation $l_{t}^{o}$ $=l_{s} \int|f(\theta)|^{2} d \theta / \int|f(\theta)|^{2}(1-\cos \theta) d \theta$, where $f(\theta)$ is the scattering amplitude of a single scatterer and can be obtained analytically. We find that $l_{t}^{o}=2.04 l_{s}, 2.2 l_{s}$, and $1.05 l_{s}$ for cases (a), (b), and (c), respectively. These numerical results confirm that the states inside the complete gaps are strongly localized with localization lengths comparable to the mean free paths and they are all of the order of the lattice constant. It should be pointed out that $l_{t}^{o}$ denotes the bare transport mean free path, which is free of interference effects. In the localized regime, the true transport mean free path should be renormalized to zero.

To simulate the CBS cone, we use samples that have a thickness $L$ a few times longer than the localization length, so that a converging result is reached. In order to separate the backscattering intensity from the specular contributions due to a residual photonic band-gap effect, we tilt the sample by $5^{\circ}$ relative to the incident wave front and calculate the farzone scattered intensity in the reflection geometry as a function of the angle. The results of 2000 configurations are shown in Figs. 3(a)-3(c) with dotted curves for the cases (a)-(c) described above, respectively. The values of $L$ chosen are $9 a$ for (a) and (b) and $18 a$ for (c). The rounded peaks at $-5^{\circ}$ represent the CBS cones from localized states, whereas

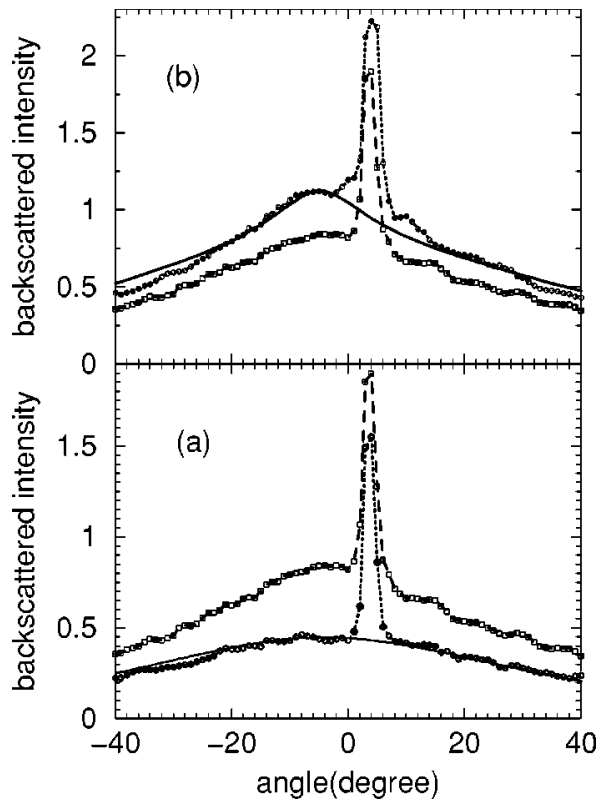

FIG. 4. Comparison between theory (the solid lines) and simulations (the dotted lines) of the CBS cones for the case $R=0.3 a$ and $\omega a / 2 \pi c=0.48$ with (a) absorption $\left(\epsilon^{\prime \prime}=0.5\right)$ and (b) amplification $\left(\epsilon^{\prime \prime}=-0.03\right)$ included. The dashed lines represent the simulation results in the absence of absorption/amplification.

the sharp peaks at $5^{\circ}$ are due to the specular contributions not related to CBS. It should be pointed out that instead of tilting the sample to avoid the specular contributions, an alternative way is to calculate the specular contributions to the total reflected intensity. This may be done by evaluating the coherent part of the reflected intensity as a function of angle, i.e., $|\langle E\rangle|^{2}$. In the study of ballistic to diffusive transition, this coherent part represents the ballistic transport and is normally separated from the diffusive part in the total transmitted intensity. ${ }^{23-25}$

Following Ref. 12, we quantify the degree of rounding in each case by extrapolating two wings in the CBS cone into a triangular cone as shown in the inset of Fig. 3(d) for case (a). The results of rounding angle $\Delta \theta$ are plotted as a function of $\lambda / \xi$ in Fig. 3(d). A straight line indicates that $\Delta \theta \rightarrow 0$ as $\xi$ $\rightarrow \infty$ at the Anderson localization transition. It is interesting to see that even in such a strongly localized regime, the CBS cone is still a useful quantity to measure and the rounding of the CBS cone can give information on the localization length of the system.

To study the effects due to absorption or amplication, we introduce an imaginary dielectric constant for each cylinder, i.e., $\epsilon=11.4+i \epsilon^{\prime \prime}$. Taking the case (a) $(R=0.30$ and $f$ $=0.48)$ as an example, the CBS cones with absorption $\left(\epsilon^{\prime \prime}\right.$ $=0.5)$ and amplification $\left(\epsilon^{\prime \prime}=-0.03\right)$ included are shown in Figs. 4(a) and 4(b) with the dotted curves, respectively. In order to make a comparison with the CBS cone without absorption/amplification, we replot the simulation results shown in Fig. 3(a) as dashed lines in Figs. 4(a) and 4(b). It is clear that the CBS cone is reduced (enhanced) in the presence of absorption (amplification) just as it is in a diffusive medium. However, the degree of rounding shifts in the opposite way. It is worth mentioning that when the amplifica- 


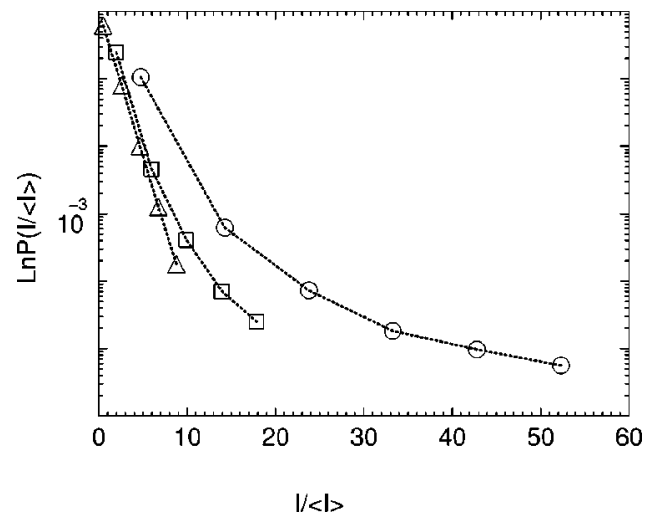

FIG. 5. The distribution of the normalized intensity of the reflected speckles for $\epsilon^{\prime \prime}=0$ (triangles), -0.03 (squares), and -0.3 (circles).

tion is larger than $\epsilon^{\prime \prime}=-0.03$, we find large angular intensity fluctuations as observed in "random lases" due to the enhancement of recurrent scattering. ${ }^{26,27}$ In order to show these fluctuations, in Fig. 5 we plot in semilog scale the distribution of the normalized speckle intensity at angles $15^{\circ}<|\theta|$ $<25^{\circ}$ for three different values of $\epsilon^{\prime \prime}$ : 0 (triangles), -0.03 (squares), and -0.3 (circles). In a passive medium, the distribution obeys Rayleigh statistics as predicted by the random matrix theory. ${ }^{5}$ In the presence of gain, a tail in the exponential decay is found, which increases with the gain parameter. Thus at large gain, it becomes diffucult to obtain a smooth CBS cone.

\section{THEORY}

In the following, we present a theory to describe the structure of CBS cones in the strong localization regime with absorption/amplification included. In a diffusive regime, the backscattering intensity can be described by the sum of three bistatic scattering coefficients, $\gamma_{s}\left(\mu_{s}, \mu_{i}\right), \gamma_{l}\left(\mu_{s}, \mu_{i}\right)$ and $\gamma_{c}\left(\mu_{s}, \mu_{i}\right)$, which represent the contribution from single scattering, diffusive transport, and interference between counterpropagating waves, respectively. ${ }^{6,7}$ Here $\mu_{i, s}$ $\equiv \cos \theta_{i, s}$, and $\theta_{i}$ and $\theta_{s}$ denote the incident and scattered angles relative to the surface normal, respectively. To be more precise, we have $\theta_{i}=0$ for normal incident light and $\theta_{s}=0$ when the backscattered light is directly opposite to the incident light. It is easy to show that by including the singleparticle scattering anisotropy, $\gamma_{s}\left(\mu_{s}, \mu_{i}\right)$ has the form

$$
\gamma_{s}\left(\mu_{s}, \mu_{i}\right)=\frac{2 \pi \bar{l}\left|f\left(\pi-\theta_{i}-\theta_{s}\right)\right|^{2}}{l_{s} \int_{0}^{2 \pi}|f(\theta)|^{2} d \theta}\left(\frac{\mu_{s}}{\mu_{s}+\mu_{i}}\right) .
$$

However, in a strong localization regime, the effects due to wave localization should be included in the evaluation of $\gamma_{l}\left(\mu_{s}, \mu_{i}\right)$ and $\gamma_{c}\left(\mu_{s}, \mu_{i}\right)$. Here, we assume that wave intensity follows a diffusive behavior with a Boltzmann diffusion constant $D_{o}=c l_{t}^{o} / d$ in a region $r<\xi$ centered at the source and decays exponentially when $r>\xi$, i.e, $\exp (-r / \xi)$. Here $d$ denotes the dimensionality of the system. This as- sumption becomes invalid when the system is close to the localization transition where $\xi \gg l_{t}^{o}$. In this case, a scaledependent diffusion constant due to interference should be used in the region between $l_{t}^{o}$ and $\xi{ }^{10}$ However, in the case of the strong localization limit considered here, we have $\xi$ $\approx l_{t}^{o}$. Therefore there does not exist a scaling region that necessitates the renormalization of the diffusion constant. This assumption allows us to replace the diffusion pole 1/ $\left(-i \omega+D_{o} q^{2}\right)$ by $1 /\left[-i \omega+D_{o}\left(q^{2}+\xi^{-2}\right)\right]$, which, in the static limit, produces a correct exponential decay in the intensity distribution at distances $r>\xi$. In the time domain, such a replacement makes the incoherent part of the timeresolved abedo to decrease like $t^{-d / 2}$ for $t<t_{c}=\xi^{2} / D_{o}$ and cross over to an exponential decay when $t>t_{c}$, i.e., $\exp \left(-t / t_{c}\right)$. If we define the time-dependent diffusion constant by using $\left\langle r^{2}\right\rangle \sim t D(t)$, we find that $D(t)=D_{o} \exp$ $\left(-t / t_{c}\right)$. A similar approach has been suggested in Ref. 10, except that instead of a smooth change, a sudden cutoff of the diffusion pole is proposed for $q<\xi^{-1}$ and the diffusion constant is assumed to be zero for $q<\xi^{-1}$ or $t>t_{c}$. With our modified diffusion pole, simple expressions for $\gamma_{l}$ and $\gamma_{c}$ can be obtained following a procedure similar to that described in Ref. 7. A brief derivation is given in the Appendix for a semi-infinite scattering medium in two dimensions (2D) $(d$ $=2$ ). The results are

$$
\begin{aligned}
\gamma_{l}\left(\mu_{s}, \mu_{i}\right)= & \frac{1}{\mu_{i} \bar{l}^{2}} \int_{0}^{\infty} \int_{0}^{\infty} \Gamma\left(z_{1}, z_{2} ; q_{\perp}=0\right) \\
& \times \exp \left[-\frac{1}{\bar{l}}\left(\frac{z_{1}}{\mu_{s}}+\frac{z_{2}}{\mu_{i}}\right)\right] d z_{1} d z_{2},
\end{aligned}
$$

$$
\begin{aligned}
\gamma_{c}\left(\mu_{s}, \mu_{i}\right)= & \frac{1}{\mu_{i} \bar{l}^{2}} \int_{0}^{\infty} \int_{0}^{\infty} \Gamma\left(z_{1}, z_{2} ; q_{\perp}\right) \\
& \times \cos \left[k_{0}\left(\mu_{i}-\mu_{s}\right)\left(z_{1}-z_{2}\right)\right] \\
& \times \exp \left[-\frac{1}{2 \bar{l}}\left(\frac{1}{\mu_{s}}+\frac{1}{\mu_{i}}\right)\left(z_{1}+z_{2}\right)\right] d z_{1} d z_{2}
\end{aligned}
$$

with

$$
\begin{aligned}
\Gamma\left(z_{1}, z_{2} ; q_{\perp}\right)= & \frac{1}{2 \pi l_{t}^{o}} \int_{0}^{\infty}\left[K_{0}\left(\frac{\sqrt{y^{2}+\left(z_{1}-z_{2}\right)^{2}}}{\eta}\right)\right. \\
& \left.-K_{0}\left(\frac{\sqrt{y^{2}+\left(z_{1}+z_{2}+2 l_{e}\right)^{2}}}{\eta}\right)\right] \cos \left(q_{\perp} y\right) d y
\end{aligned}
$$

and

$$
\eta^{-2}=\xi^{-2} \pm\left(\frac{l_{t}^{o} l_{a, g}}{2}\right)^{-1}
$$

where $K_{0}$ is the modified Bessel function of the second kind, $k_{0}=\omega / c, q_{\perp}=k_{0}\left(\sin \theta_{s}+\sin \theta_{i}\right)$, and extrapolation length $l_{e}$ $=\left(0.78 l_{t}^{o}\right)(1+R) /(1-R)$. Here $R$ is the ratio of the incoming flux to the outgoing flux at the sample boundary. It 
should be pointed out that in Eq. (4), a constant bare extrapolation length $l_{e}$ has been used for the entire $q$ space. It has been suggested in Ref. 10 that a $q$-dependent renormalized extrapolation length, similar to the renormalization of the diffusion constant, should be used in the critical region $\xi^{-1}$ $<q<\left(l_{t}^{o}\right)^{-1}$. Since we have $\xi \approx l_{t}^{o}$ in the strong localization region, we can use a bare extrapolation length $l_{e}$ for the entire region of $q>\xi^{-1}$. As for the localized part with $q$ $<\xi^{-1}$ or $r>\xi$, we assume it gives a negligible contribution to $\gamma_{l}$ and $\gamma_{c}$ when compared with the diffusive part. Thus, the error we introduced is expected to be insignificant and the question of extrapolation length renormalization becomes unimportant. The justification of this assumption will be discussed later.

The presence of the last term in Eq. (5) represents the interplay between absorption /gain and diffusion and is derived in Eq. (A12) of the Appendix with the assumption of $l_{a, g} \gg l_{t}^{0}$, where $l_{a}$ and $l_{g}$ are, respectively, the absorption and gain lengths of the medium. ${ }^{7}$ This result can also be understood intuitively by using some diffusion arguments, i.e., $L_{a, g}^{2}=D_{0} \tau_{a, g}, D_{0}=l_{0}^{t} c / d$, and $\tau_{a, g}=l_{a, g} / c$, where $L_{a, g}$ and $\tau_{a, g}$ are the decay (growth) length and absorption (gain) time, respectively. ${ }^{1}$ The effects due to wave localization come from the presence of the $\xi^{-2}$ term in our modified diffusion pole. This simple insertion is to cut off the diffusion pole to take into account the wave localization. It does not include the interplay between absorption/gain and localization. However, the contribution to the change of the CBS cone from the interaction between absorption/gain and localization can be assumed to be much smaller than that from the diffusion part due to the exponential decay in the intensity when $q<\xi^{-1}$. In this case, the diffusion pole is dominated by the large term $\xi^{-2}$, which cuts off the contribution to the CBS cone and makes the error insignificant. This assumption is supported by a recent work on the absorption in a metallic photonic crystal. ${ }^{28}$ It has been found that the absorption is much smaller in the gap region than in the band region due to the evanescent decay of wave intensity in the gap region. Although the last term in Eq. (5) does not include the interaction between localization and absorption/gain, the presence of this term is effectively to enhance (or reduce) the localization effect in the case of absorption (or gain) if we consider $\eta$ as an effective localization length. This in turn will reduce (or enhance) the CBS cone as we have seen in the numerical simulations (Fig. 4).

In the absence of absorption or amplification, where $\bar{l}$ $=l_{s}$, we use numerically calculated $l_{s}, l_{t}^{o}, \xi$, and $f(\theta)$ in Eqs. (1)-(5). The effective medium approximation is used to estimate $R$. The results are shown as the solid curves in Figs. 3(a)-3(c). Apart from some small statistical fluctuations, we have obtained excellent quantitative agreements between theory and simulations for a wide range of angles in all cases without any adjustable parameter. To isolate the CBS effect, we also plot separately the terms $\gamma_{s}, \gamma_{l}$, and $\gamma_{c}$ in Fig. 6 . Compared with the same term in the diffusive regime, $\gamma_{c}$ now covers a much wider range of angles. Appreciable amount of intensity appears even when the scattered angle $\theta_{s}$ is above $10^{\circ}$. From the three curves of $\gamma_{s}\left(\theta_{s}\right)$ shown in Fig.

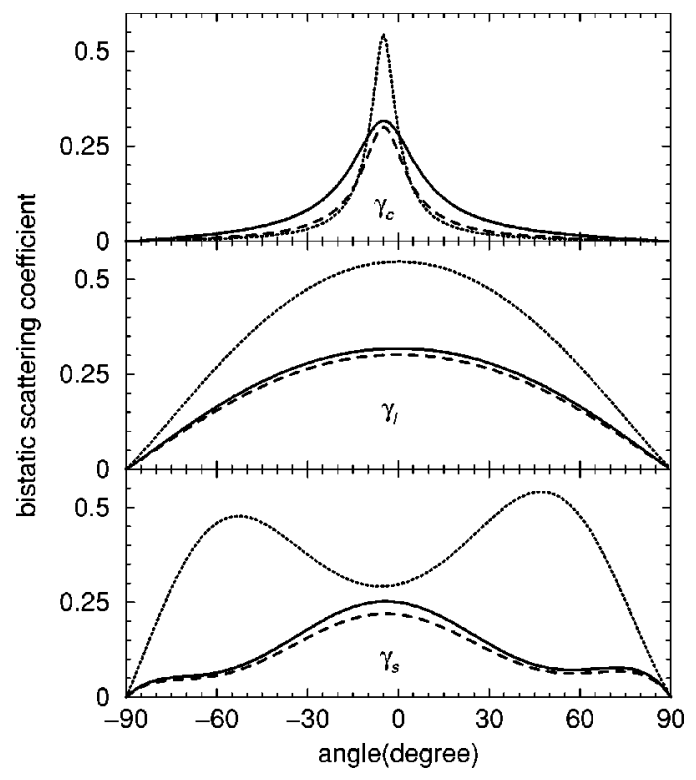

FIG. 6. Comparison of $\gamma_{s}, \gamma_{l}$, and $\gamma_{c}$ for the three cases shown in Fig. 2: (a) in solid lines, (b) in dashed lines, and (c) in dotted lines.

6 , it is easy to see that when $\theta_{s}>10^{\circ}, \gamma_{s}$ shows strong angular dependence and behaves very differently between cases (a), (b), and (c). This is due to large angular dependence in the scattering amplitude $f$ and its sensitivity to the ratio $\lambda / \sqrt{\epsilon} R$ near Mie resonance. In fact, the ratio of $\lambda / \sqrt{\epsilon} R$ is about 2 for the cases (a) and (b) and 1.5 for the case (c). If we ignore the single scattering anisotropy, Eq. (1) reduces to the known result of $\gamma_{s}\left(\mu_{s}, \mu_{i}\right)=\mu_{s} /\left(\mu_{s}+\mu_{i}\right)$ in the absence of absorption/amplification. ${ }^{7}$ By using this relation in our theoretical calculations, we replot the results of cases (a) and (c) as dashed curves in Figs. 7(a) and 7(b). Compared to the results of Eq. (1) (solid lines), appreciable discrepancies between theory and simulations appear in the wings of CBS cones when $\theta_{s}>10^{\circ}$. Thus, in a strong localization regime, it is necessary to include the single scattering anisotropy in Eq. (1) in order to achieve a quantitative agreement with the simulation data.

In the presence of absorption or amplification, we also calculate the extinction length $\bar{l}$ numerically for case (a) shown in Fig. 3. The absorption and gain lengths are found to be $l_{a} \approx 10 l_{s}$ for $\epsilon^{\prime \prime}=0.5$ and $l_{g} \approx 80 l_{s}$ for $\epsilon^{\prime \prime}=-0.03$, respectively. By using these numbers in Eqs. (1)-(5), we obtain two solid curves in Fig. 4. The excellent agreements between theory and simulations show the validity of Eqs. (1) - (5) even in the presence of absorption or amplification.

The generalization of Eqs. (1)-(5) to 3D systems is straightforward. In this case, the function $\Gamma$ in Eq. (4) should be replaced by

$$
\begin{aligned}
\Gamma\left(z_{1}, z_{2} ; q_{\perp}\right)= & \frac{3}{2 l_{t}^{o}}\left[\frac{\exp \left(-\left|z_{1}-z_{2}\right| \sqrt{\eta^{-2}+q_{\perp}^{2}}\right)}{\sqrt{\eta^{-2}+q_{\perp}^{2}}}\right. \\
& \left.-\frac{\exp \left(-\left|z_{1}+z_{2}+2 l_{e}\right| \sqrt{\eta^{-2}+q_{\perp}^{2}}\right)}{\sqrt{\eta^{-2}+q_{\perp}^{2}}}\right]
\end{aligned}
$$




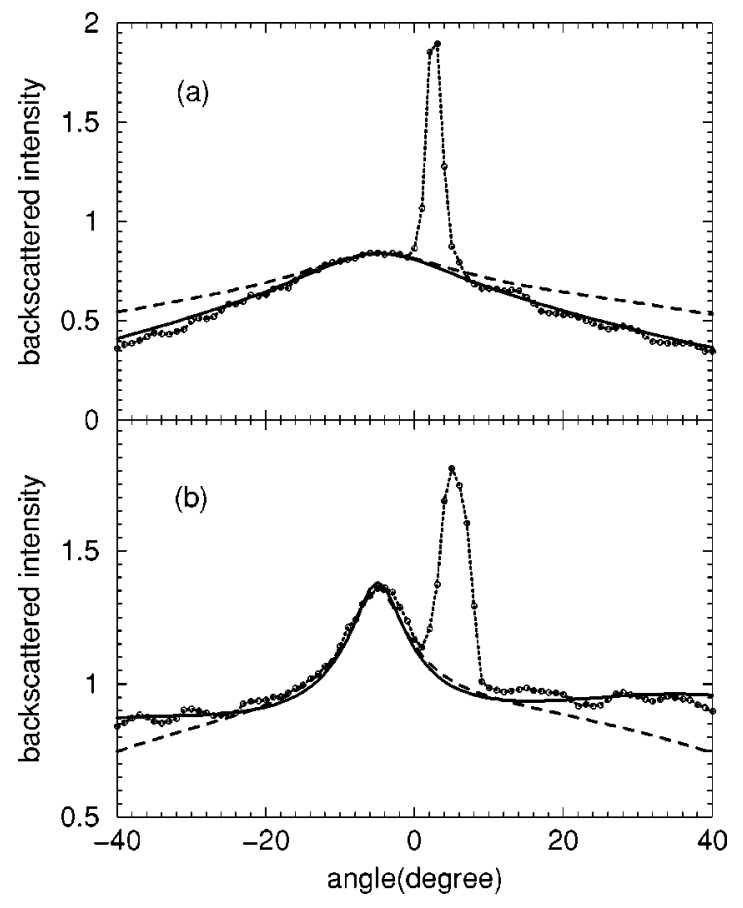

FIG. 7. The CBS cones for two cases: (a) $R=0.3 a$ at $\omega a / 2 \pi c$ $=0.48$ and (b) $R=0.32 a$ at $\omega a / 2 \pi c=0.64$ with the single scattering anisotropy included (solid and dotted lines) and without the single scattering anisotropy (dashed lines), respectively. The dotted lines represent the simulation result.

with

$$
\eta^{-2}=\xi^{-2} \pm\left(\frac{l_{t}^{o} l_{a, g}}{3}\right)^{-1}
$$

where $\quad q_{\perp}=k_{0}\left[\left(\sin \theta_{i} \cos \psi_{i}+\sin \theta_{s} \cos \psi_{s}\right)^{2}+\left(\sin \theta_{i} \sin \psi_{i}\right.\right.$ $\left.\left.+\sin \theta_{s} \sin \psi_{s}\right)^{2}\right]^{1 / 2}$ and $i$ and $s$ stand for the incident and scattered angles, respectively. ${ }^{7}$ Again, Eqs. (6) and (7) are valid only in the strong localization regime.

\section{DISCUSSION AND CONCLUSIONS}

In our theory, we have assumed that the diffusive part of the wave propagation represents the dominant contribution to the CBS cone, whereas the localized part makes a negligible contribution due to its exponential decay in wave intensity. This assumption is supported by the excellent agreement between theory and simulation for all the cases we have studied without any adjustable parameters. In fact, our assumption is also supported by the result of the random matrix theory, which predicts Rayleigh-type statistics in the reflected intensity distribution even in the localized regime. ${ }^{5}$ If the contribution from the localized part were not negligible, one should expect a tail in the exponential decay similar to the case of transmitted intensity distribution. ${ }^{1}$ Certainly, this is a unique feature of wave propagation in the reflection geometry, not shared by the transmitted waves.

At this juncture, we would like to stress that the 2D disorder photonic crystals we have considered here are completely random. Thus, all states are localized, including those in the passbands. However, due to the hard-core repulsions among the scatterers, systems possess certain short-range order even when they have been completely randomized. As a result of such short-range order as well as the the presence of Mie resonances, some quantitative differences in the transport properties between the states in the gaps and in the bands still remain. For instance, the localization length inside a gap is generally smaller than that in a band. In fact, the role played by resonant scattering in determining the band-gap position has been studied. ${ }^{29,30}$ Since the focus of this work is in the strong localization regime, we did not study the CBS cone arising from those less localized states in a band. However, both the method of numerical simulation and analytical theory presented here can also be applied to the localized states in a band.

Instead of complete randomness, if only a small disorder is introduced, the wave propagation inside a gap remains evanescent in nature. In return, the statistics of reflected speckle intensities will exhibit a non-Rayleigh distribution. ${ }^{31}$ In this case the analytical theory presented here cannot apply. However, for the case of complete randomness studied here, the second and third gaps shown in Fig. 3 are closed. Waves lose their evanescent nature completely and Rayleigh statistics are recovered. ${ }^{31}$ This makes the analytical theory valid. The qualitative difference in the transport behavior between the cases of small randomness and large randomness has first been reported in the study of $1 \mathrm{D}$ disordered photonic crystals. $^{32,33}$ It has been found there can exist two types of localized states inside a band gap. In the case of large disorder, all the states inside a gap belong to normal-type localized states, satisfying the single-parameter scaling theory. However, when disorder is small, the localized states well inside a gap do not satisfy the single-parameter scaling theory due to the residual evanescent nature of the waves. Such distinct transport behaviors inside a gap between the cases of small and large disorder also appear in higher dimensions. In this work, our focus is on systems with large disorder. The structure of the CBS cone arising from the localized states inside the gap of a weakly disordered photonic crystal remains an interesting subject for future study.

We would also like to point out that in the diffusive part, although the number of scatterings is small in the strong localization regime, nevertheless, this small number of scatterings is sufficient to develop diffusive behavior. If we take a typical case of $\xi \approx 3 l_{0}^{t}$ and use $D_{0}=\left(l_{0}^{t}\right)^{2} / 3 t_{0}$, where $t_{0}$ is the scattering time, the crossover time it takes from diffusive to localized behavior is about $t_{c}=\xi^{2} / D_{0} \approx 27 t_{0}$. Thus, it takes about 27 scattering processes before waves become localized. In a recent study of ballistic to diffusive transition using transmitted pulse measurements, it has been found that the peak of the pulse starts to develop a diffusive behavior when the sample thickness is as small as three mean free paths. ${ }^{25}$. This indicates that only a few scattering processes are needed to develop a diffusive behavior. Therefore, even in a strong localization regime, it is possible to have a diffusive region that constitutes the dominant part of wave propagation in the reflection geometry.

Although our present work is focused on the strong localization regime, both the numerical and theoretical approaches presented here can, in principle, be extended to 
include the critical region. Numerically, one has to simulate the CBS cone arising from states with $\xi \gg l_{0}^{t}$. In this case, the sample size becomes very large, i.e., $W \gg L>\xi$. Unfortunately, our present computing capability does not permit us to perform such large-scale simulation. Analytically, as the critical region is approached, one has to renormalize the diffusion constant as well as the extrapolation length as suggested in Ref. 10. The interplay between absorption/ amplifcation and diffusion can also be obtained from the intuitive arguments described earlier by adopting a scaledependent diffusion constant $D(L)=D_{0} l_{0}^{t} / L$ to replace $D_{0}$ for $L<\xi$. Such a replacement will lead to the result of $L_{a, g}^{2}$ $=\left[\left(l_{0}^{t}\right)^{2} l_{a, g} / d\right]^{2 / 3} \cdot{ }^{34,35}$ The existence of the factor $\xi^{-2}$ in the diffusion pole will again provide a smooth cutoff when $L$ $>\xi$. Of course, the validity of this generalization should be tested against the $a b$ initio simulation as we have done here. If this can be done, through comparison between theory and simulation, it may be able to resolve the controversial issue of extrapolation length renormalization in the critical region.

The excellent agreement in the structure of the CBS cone between theory and simulations suggests the possibility of a method to determine the localization length even in the presence of absorption. In order to do so, the absorption length and the scattering mean free path should be determined first. In order to know the absorption length of the medium, one can determine the absorption time $t_{a}$ by using a pulse measurement. ${ }^{36}$ In a thick sample, it is expected that the time-resolved total reflected intensity decays as $\exp \left(-t / t_{a}\right)$. In order to determine the scattering mean free path, one can measure the decay of ballistic (coherent) intensity as a function of sample thickness in thin samples in transmission measurements, i.e., $|\langle E\rangle|^{2} \sim \exp \left(-L / l_{s}\right){ }^{22}$ Then $l_{t}^{0}$ and the function $\gamma_{s}$ of Eq. (1) are calculated by using the scattering function $f(\theta)$ of the single scatterer. Finally, the localization length can be determined by fitting the measured CBS cone to the analytic results.

In summary, we have simulated CBS cones of light at different wavelengths and localization lengths inside the complete gap of disordered photonic crystals in two dimensions by using the multiple-scattering method. We have found that CBS cones still exist and the curve is not flat even when the localization length $\xi$ is two to three times that of the lattice constant. Furthermore, the rounding of the CBS cone is found to follow a simple relation $\lambda / \xi$, which can be used to estimate the localization length. Like in a diffusive regime, the presence of absorption or amplification is found to reduce or enhance the CBS cone. In addition to simulations, we have also presented an analytical theory that incorporates scattering and transport mean free paths, localization length, absorption/amplification length, and single-particle scattering anisotropy. We have obtained excellent quantitative agreement between theory and simulations with a wide range of angles in all the cases we have studied without any adjustable parameters. This makes it possible to determine the localization length from a measured CBS cone, even in the presence of absorption. The main results obtained here are also valid in three dimensions.

\section{ACKNOWLEDGMENTS}

We wish to thank P. Sheng and B. A. van Tiggelen for useful discussions. This work was supported by Hong Kong RGC Grants Nos. HKUST 6137/97P and 6160/99P.

\section{APPENDIX}

In this appendix, we give some key steps that lead to the final results of Eqs. (4) and (5) for the CBS cone in 2D. For convenience, the notations used here follow the 3D work of van der Mark et al. in Ref. 7. Also, we assume that wave speed $c$ in vacuum is unity. In $2 \mathrm{D}$, the single-particle scattering operator $s\left(\vec{k}_{1}, \vec{k}_{2}\right)$ takes the form ${ }^{1}$

$$
s\left(\vec{k}_{1}, \vec{k}_{2}\right)=4 i \sqrt{\frac{\pi k_{0}}{2}} \exp \left(i \frac{\pi}{4}\right) f\left(\vec{k}_{0}^{\prime}, \vec{k}_{0}\right) .
$$

Assuming a point scatterer, Eq. (A1) has the following form in real space:

$$
s_{\alpha}\left(\vec{r}_{1}, \vec{r}_{2}\right)=4 i \sqrt{\frac{\pi k_{0}}{2}} \exp \left(i \frac{\pi}{4}\right) f \delta\left(\vec{r}_{1}-\vec{r}_{\alpha}\right) \delta\left(\vec{r}_{2}-\vec{r}_{\alpha}\right)
$$

where $\vec{r}_{\alpha}$ denotes the position of the scatterer $\alpha$. From Eq. (A2), we can write the vertex function for the ladder diagrams as

$$
\begin{aligned}
L_{s} & =\sum_{\alpha} \int s_{\alpha}\left(\vec{r}_{1}, \vec{r}_{2}\right) s_{\alpha}^{*}\left(\vec{r}_{3}, \vec{r}_{4}\right) d \vec{r}_{\alpha} \\
& =\frac{4 k_{0}}{l} \delta\left(\vec{r}_{1}-\vec{r}_{2}\right) \delta\left(\vec{r}_{3}-\vec{r}_{1}\right) \delta\left(\vec{r}_{4}-\vec{r}_{1}\right),
\end{aligned}
$$

where $l=1 /\left(2 \pi n_{0} f^{2}\right)$ and $n_{0}$ is the density of scatterers. For point scatterers, the function $f$ is angular independent and the transport mean free path equals the scattering mean free path, i.e, $l_{t}(0)=l_{s}=l$. Thus, the function $F\left(\vec{r}_{1}, \vec{r}_{2}\right)$ in the ladder diagram satisfies

$$
\begin{aligned}
F\left(\vec{r}_{1}, \vec{r}_{2}\right)= & \left(4 \sqrt{\frac{\pi k_{0}}{2}} f\right)^{4} n_{0}^{2} A\left(\vec{r}_{1}-\vec{r}_{2}\right) \\
& +n_{0}\left(4 \sqrt{\frac{\pi k_{0}}{2}} f\right)^{2} \int A\left(\vec{r}_{1}-\vec{r}^{\prime}\right) F\left(\vec{r}_{1}, \vec{r}_{2}\right) d \vec{r}^{\prime},
\end{aligned}
$$

where

$$
A\left(\vec{r}_{1}-\vec{r}_{2}\right)=\left\langle G_{k_{0}+\omega / 2}\left(\vec{r}_{1}-\vec{r}_{2}\right)\right\rangle\left\langle G_{k_{0}-\omega / 2}^{*}\left(\vec{r}_{1}-\vec{r}_{2}\right)\right\rangle,
$$

and 


$$
\begin{aligned}
\left\langle G_{k_{0}}(\vec{r})\right\rangle & =\frac{1}{(2 \pi)^{2}} \int \frac{e^{i \vec{k} \cdot \vec{r}} d \vec{k}}{\left[k_{0}+i /(2 \bar{l})\right]^{2}-k^{2}} \\
& =-i H_{0}^{(1)}\left[\left\{k_{0}+i /(2 \bar{l})\right\}|\vec{r}|\right] / 4,
\end{aligned}
$$

where $1 / \bar{l}=1 / l \pm 1 / l_{a, g}$ has been defined in Sec. II and $H_{0}^{(1)}$ is the Hankle function of the first kind. Equation (A4) is solved in the momentum space by taking its Fourier transform, i.e.,

$$
F(\vec{q})=\frac{\frac{16 k_{0}^{2}}{l^{2}} A(\vec{q})}{1-\frac{4 k_{0}}{l} A(\vec{q})},
$$

where

$$
A(\vec{q})=\int\left\langle G_{k_{0}+\omega / 2}(\vec{r})\right\rangle\left\langle G_{k_{0}-\omega / 2}^{*}(\vec{r})\right\rangle e^{-i \vec{q} \cdot \vec{r}} d \vec{r}
$$

Substituting Eq. (A6) into Eq. (A8) and taking the diffusion limit, we find

$$
F(\vec{q})=\frac{8 k_{0} /\left(l \bar{l}^{2}\right)}{a-\frac{i w b}{D_{0}}+q^{2}},
$$

where

$$
a=\left\{\begin{array}{cc}
\frac{2\left(l+l_{a}\right)^{2}}{l l_{a}^{3}} & \text { for absorption } \\
\frac{-2\left(l_{g}-l\right)^{2}}{l l_{g}^{3}} & \text { for gain, }
\end{array}\right.
$$

$$
b=\left\{\begin{array}{lc}
\frac{\left(l+l_{a}\right)}{l_{a}} & \text { for absorption } \\
\frac{\left(l_{g}-l\right)}{l_{g}} & \text { for gain, }
\end{array}\right.
$$

where $D_{0}=l / 2$. In absence of absorption and gain, we have $a=0$ and $b=1$ and Eq. (A9) contains a diffusion pole as expected. In the situation $l_{a, g} \gg l, a$ and $b$ can be approximated by $\pm\left(l l_{a, g} / 2\right)^{-1}$ and $b=1$. Here + and - correspond to the absorption and gain, respectively. In the localization regime, from the assumption described in Sec. III, we should replace the factor $1 /\left(a-i \omega b / D_{0}+q^{2}\right)$ in Eq. (A9) by $1 /(a$ $\left.-i \omega b / D_{0}+q^{2}+\xi^{-2}\right)$, where $\xi$ is the localization length. In the case $l_{a, g} \gg l$, Eq. (A9) becomes

$$
F(\vec{q})=\frac{8 k_{0} /\left(l \bar{l}^{2}\right)}{-\frac{i w}{D_{0}}+\eta^{-2}},
$$

where $\eta^{-2}=\xi^{-2} \pm\left(l l_{a, g} / 2\right)^{-1}$.

Thus, in the static limit $(\omega=0)$, by taking the Fourier transform of Eq. (A12), we obtain

$$
F(\vec{r})=\frac{2 k_{0}}{\pi l \bar{l}^{2}} K_{0}\left(\frac{r}{\eta}\right)
$$

where $K_{0}$ is the modified Bessel function of the second kind. In the real situation where the function $f$ is angular dependent, the bare transport mean free path $l_{t}^{0}$ should be used for $l$. Inserting Eq. (A13) into the bistatic scattering coefficients for the ladder and maximally crossed diagrams described in Ref. 7, we obtain Eqs. (4) and (5).
${ }^{1}$ See, for example, Scattering and Localization of Classical Waves in Random Media, edited by P. Sheng (World Scientific, Singapore, 1990); P. Sheng, Introduction to Wave Scattering, Localization, and Mesoscopic Phenomena (Academic Press, New York, 1995).

${ }^{2}$ A.Z. Genack and N. Garcia, Phys. Rev. Lett. 66, 2064 (1991); 66, 1850 (1991).

${ }^{3}$ D.S. Wiersma, P. Bartolini, A. Lagendijk, and R. Righini, Nature (London) 390, 671 (1997); 398, 207 (1999).

${ }^{4}$ A.A. Chabanov, M. Stoytchev, and A.Z. Genack, Nature (London) 404, 850 (2000).

${ }^{5}$ A. Garcia-Martin, T. Lopez-Ciudad, and J.J. Saenz, Phys. Rev. Lett. 81, 329 (1998).

${ }^{6}$ Y. Kuga and A. Ishimaru, J. Opt. Soc. Am. A 8, 831 (1984); M.P. Van Albada and A. Lagendijk, Phys. Rev. Lett. 55, 2692 (1985); P.E. Wolf and G. Maret, ibid. 55, 2696 (1985). E. Akkermans, P.E. Wolf, and R. Maynard, ibid. 56, 1471 (1986); M. Kaveh, M. Rosenbluh, I. Edrei, and I. Freund, ibid. 57, 2049 (1986).

${ }^{7}$ M.B. van der Mark, M.P. van Albada, and A. Lagendijk, Phys. Rev. B 37, 4336 (1988); E. Akkermans, P.E. Wolf, R. Maynard, and G. Maret, J. Phys. (France) 49, 77 (1988); F.C. MacKintosh and S. John, Phys. Rev. B 37, 1884 (1988).

${ }^{8}$ D.S. Wiersmai, M.P. van Albada, and A. Lagendijk, Phys. Rev. Lett. 75, 1739 (1995); Phys. Rev. E 54, 4256 (1996).

${ }^{9}$ A.Yu. Zyuzin, Europhys. Lett. 26, 517 (1994); S.C. Feng and Z.Q. Zhang, Phys. Rev. B 54, 3240 (1996); W. Deng, D.S. Wiersma, and Z.Q. Zhang, ibid. 56, 178 (1997).

${ }^{10}$ R. Berkovits and M. Kaveh, Phys. Rev. B 36, 9322 (1987).

${ }^{11}$ I. Edrei and M.J. Stephen, Phys. Rev. B 42, 110 (1990).

${ }^{12}$ F.J.P. Schuurmans, M. Megens, D. Vanmaekelbergh, and A. Lagendijk, Phys. Rev. Lett. 83, 2183 (1999).

${ }^{13}$ B.A. van Tiggelen, A. Lagendijk, and D.S. Wiersma, Phys. Rev. Lett. 84, 4333 (2000).

${ }^{14}$ S. John, Phys. Rev. Lett. 58, 2486 (1987).

15 See, for example, Photonic Band Gaps and Localization, edited by C.M. Soukoulis (Plenum, New York, 1993); J.D. Joannopolous, R.D. Meade, and J.N. Winn, Photonic Crystals (Princeton University, Princeton, 1995).

${ }^{16}$ R. Dalichaouch, J.P. Armstrong, S. Schultz, P.M. Platzman, and S.L. McCall, Nature (London) 354, 53 (1991).

${ }^{17}$ Z.Q. Zhang, C.C. Wong, K.K. Fung, Y.L. Ho, W.L. Chan, S.C. 
Kan, T.L. Chan, and N. Cheung, Phys. Rev. Lett. 81, 5540 (1998).

${ }^{18}$ Yu.A. Vlasov, M.A. Kaliteevski, and V.V. Nikolav, Phys. Rev. B 60, 1555 (1999).

${ }^{19}$ A.F. Koenderink, M. Megens, G. van Soest, W.L. Vos, and A. Lagendijk, Phys. Lett. A 268, 104 (2000).

${ }^{20}$ L.M. Li and Z.Q. Zhang, Phys. Rev. B 58, 9587 (1998).

${ }^{21}$ G. Cwilich and M.J. Stephen, Phys. Rev. B 35, 6517 (1987).

${ }^{22}$ H.P. Schriemer, M.L. Cowan, J.H. Page, P. Sheng, Z. Liu, and D.A. Weitz, Phys. Rev. Lett. 79, 3166 (1997).

${ }^{23}$ A. Garcia-Martin, J.J. Saenz, and M. Nieto-Vesperinas, Phys. Rev. Lett. 84, 3578 (2000).

${ }^{24}$ A.A. Chabanov and A.Z. Genack, Phys. Rev. E 56, R1338 (1997).

${ }^{25}$ Z.Q. Zhang, I.P. Jones, H.P. Schriemer, J.H. Page, D.A. Weitz, and P. Sheng, Phys. Rev. E 60, 4843 (1999).

${ }^{26}$ H. Cao, Y.G. Zhao, S.T. Ho, E.W. Seelig, Q.H. Wang, and R.P.H. Chang, Phys. Rev. Lett. 82, 2278 (1999); 84, 5584 (2000).
${ }^{27}$ D.S. Wiersma, M.P. van Albada, B.A. van Tiggelen, and A. Lagendijk, Phys. Rev. Lett. 74, 4193 (1995).

${ }^{28}$ L.M. Li, Z.Q. Zhang, and X. Zhang, Phys. Rev. B 58, 15589 (1998).

${ }^{29}$ M. Kafesaki and E.N. Economou, Phys. Rev. B 52, 13317 (1995).

${ }^{30}$ E. Lidorikis, M.M. Sigalas, E.N. Economou, and C.M. Soukoulis, Phys. Rev. Lett. 81, 1405 (1998).

${ }^{31}$ X. Zhang and Z. Q. Zhang (unpublished).

${ }^{32}$ L.I. Deych, D. Zaslavsky, and A.A. Lisyansky, Phys. Rev. Lett. 81, 5390 (1998).

${ }^{33}$ L.I. Deych, A.A. Lisyansky, and B.L. Altshuler, Phys. Rev. Lett. 84, 2678 (2000); Phys. Rev. B 64, 224202 (2001).

${ }^{34}$ P.W. Anderson, Philos. Mag. B, 52, 505 (1985).

${ }^{35}$ R. Berkovits and M. Kaveh, J. Phys. C 2, 307 (1990).

${ }^{36}$ J.H. Page, H.P. Schriemer, A.E. Bailey, and D.A. Weitz, Phys. Rev. E 52, 3106 (1995). 